\title{
Análisis Funcional de la Conducta Verbal del Terapeuta en el Tratamiento de un Caso de Ansiedad
}

\section{Functional Analysis of the Therapist's Verbal Behavior in the Treatment of an Anxiety Case}

\author{
Andrea López Bosch y Ricardo de Pascual Verdú \\ Instituto Terapéutico Madrid, España
}

\begin{abstract}
Resumen. En el presente artículo se presenta un análisis funcional de la conducta verbal del terapeuta en un caso típico de ansiedad. Una vez catalogadas las verbalizaciones terapéuticas a reforzar y las antiterapéuticas a extinguir, se analiza hasta qué punto las verbalizaciones del terapeuta han ejercido un control en el sentido deseado por éste para modificar la conducta verbal del cliente. Para ello, se observaron cuatro sesiones grabadas de las quince que constituyeron el tratamiento completo, y se registró la ocurrencia de las cadenas funcionales deseadas. Tras esta observación, se concluyó que la acción del terapeuta es coherente con lo esperado al modelar la interacción terapéutica como un proceso de moldeamiento verbal.
\end{abstract}

Palabras clave:análisis funcional, conducta verbal, estudio de procesos, ansiedad.

\begin{abstract}
In this paper, a functional analysis of the therapist's verbal behavior in a typical anxiety case is presented. Once all therapeutic utterances to be reinforced and anti-therapeutic utterances to be extinguished were listed, the extent to which the therapist's utterances had played a role in effecting the desired change in the client's verbal behavior was analyzed. In order to do so, four of the fifteen recorded sessions from the treatment were observed, and the occurrence of the desired operant chains was noted. After this, it was concluded that the therapist's performance is consistent with its aims, in that the clinical interaction is modeled as a verbal shaping process.

Keywords: functional analysis, verbal behavior, process research, anxiety.
\end{abstract}

\section{Introducción}

Se trabajó con R. que es una mujer de 33 años. Acude a tratamiento por un problema de ansiedad. Siente mareos, malestar estomacal, dolores de cabeza y nerviosismo en ciertas situaciones. Al estudiar este caso quisimos centrarnos en el análisis de manejo de contingencias verbales por parte del terapeuta.

Durante toda su vida R. dice haber sido propensa

La correspondencia sobre este artículo debe enviarse indistintamente a los correos r.pascual.verdu@gmail.com o andrea.lopezbosch@ gmail.com a unos leves síntomas febriles, especialmente durante algunas fases del ciclo menstrual. Ha acudido a muchos especialistas distintos para ir descartando dolencias somáticas que pudieran estar detrás de este conjunto de síntomas, no encontrando éstos ninguna causa subyacente más allá de una mayor vulnerabilidad inmunológica y una leve anemia. Meses antes de acudir a terapia psicológica sufrió dos episodios que ella califica de "ataques de ansiedad" que le hicieron sospechar de una posible naturaleza psicológica del problema por su contigüidad con cambios en su entorno. El inicio de esta sospecha coincide con un agravamiento de los síntomas, que se 
vuelven progresivamente más incapacitantes. Toma medicación preventiva para la migraña y ocasionalmente un antihistamínico ligero para dormir.

En el momento de acudir a sesión, R. ha dejado de hacer muchas de las cosas que hacía para entretenerse, como viajes, salir con amigos o su pareja, etc., y llora frecuentemente. Además, un leve miedo a la oscuridad que siempre había estado presente se ha agravado también, teniendo que dormir con la persiana ligeramente levantada. De la misma manera, dice haber desarrollado cierta aprensión a la ducha, que ha pasado de ser un momento relajante a algo que hace lo más rápido posible por el nerviosismo que le produce.

Estudió empresariales y trabaja en un banco. Se casó en septiembre de 2008 con su novio, tras ocho años de relación. Dice no tener problemas para hacer o mantener amistades, y su relación con su familia y pareja es buena. Su marido es de otra ciudad, y constantemente se queja sobre la vida en su lugar de residencia actual, lo cual es fuente de angustia para R., que considera que eventualmente irán a vivir a la ciudad de su marido, pero "no ahora". En cualquier caso, ambos viajan con mucha frecuencia a esa ciudad para visitar a la familia de su marido y a los amigos que ambos tienen allí. R. reconoce que esto es "un sacrificio" para ella, que tiene que emplear buena parte de su tiempo libre de la semana en preparar la maleta e invertir gran parte del fin de semana en el viaje.

Comenzó a trabajar en 2003, momento en que ella considera que los síntomas a los que siempre fue propensa comenzaron a agravarse. Sin embargo, es en 2008, coincidiendo con un cambio de oficina, cuando la frecuencia en la que tiene que pedir unos días por estar enferma comienza a aumentar. La presión y volumen de trabajo en esta nueva oficina eran sensiblemente mayores que en la anterior. Un poco después comenzó a trabajar en la oficina una directora nueva que le resultaba desagradable en su trato, aumentando aún más la sensación de presión. En diciembre de 2009 se mudó junto con su marido a otro piso en el extrarradio, lo que aparejó, como es habitual en esos casos, muchos cambios y presiones diferentes. R. dice haber estado enferma prácticamente todo el invierno de 2009. Durante ese año pidió frecuentemente bajas por enfermedad y faltó al trabajo en numerosas ocasiones. Muchas de estas ocasiones coincidían con finales de vacaciones o puentes. Además sufrió un atraco a mano armada en su puesto de trabajo que, aunque no tuvo consecuencias importantes, fue -como es lógico- una experiencia tremendamente desagradable.

Es en junio de 2010 cuando se da el primer episodio de ansiedad. Un viernes, al volver de trabajar, comenzó a sentir ahogo y mareos en el salón de su casa. Se asomó a la ventana para atenuar la sensación de asfixia, pero no tuvo mucho éxito. Le daba miedo cerrar los ojos para dormir, y sentía que la habitación "se le caía encima". Los síntomas finalmente se disminuyeron, aunque dijo no poder estar tranquila en su casa durante el fin de semana. No salió más que a dar un breve paseo con su marido, y recuerda haber vivido con aprensión tanto el salir a la calle como sobre todo el volver a casa. En la siguiente semana, faltó tres días al trabajo. Todo el tiempo se sentía culpable y preocupada por si podrían o no hacer un viaje al extranjero que tenían planeado. Finalmente pudieron hacerlo y ella lo disfrutó enormemente, sin sentirse en ningún momento mal mientras duró su estancia allí.

El segundo episodio se dio también un viernes al volver a casa, a mediados de julio del mismo año. Se tomó un antihistamínico ligero para facilitar el sueño y tampoco salió de casa más que a dar un breve paseo. También en esta ocasión tenían planeado un viaje, aunque dentro del territorio nacional.

Desde entonces, ha reducido drásticamente sus salidas de ocio y ve con menos frecuencia a sus amigos. Cada vez que va a preparar un viaje a la ciudad de su marido o a otro sitio pasa los días previos agobiada por los preparativos y preguntándose si podrá ir o no, y si "le dará otra vez". Varias veces al día tiene momentos en los que se encuentra mal y comienza a preguntarse "¿Por qué me pasa esto a mí?" y cosas similares.

La demanda de R. es la disminución de su malestar y la recuperación de su estilo de vida previo al problema.

Con los datos obtenidos se pudo realizar el siguiente análisis funcional:

El papel de las variables disposicionales es fundamental para comprender por qué y cómo ciertas cadenas funcionales se han dado en lugar de otras. 
En el caso de R., tras la fase de evaluación, se consideraron las siguientes variables disposicionales como relevantes:

- Cambio relativamente reciente de domicilio: al mudarse, los discriminativos del entorno que previamente permitían a $\mathrm{R}$. obtener una tasa de refuerzos más elevada que la actual han desaparecido. El nuevo trabajo de $\mathrm{R}$. le consume más tiempo, impidiendo a su vez que condicione nuevos discriminativos que faciliten el refuerzo, esto es, que aparezcan situaciones en su vida cotidiana que faciliten la conducta adaptativa. Asimismo, al no existir asociaciones previas sólidas, es más fácil que se condicione con estímulos nuevos y salientes (sean estos aversivos o apetitivos). Además, la distancia al lugar donde trabaja favorece que pase al día mucho tiempo en el coche o transporte público.

- Cambio de puesto de trabajo: el aumento de demanda en sus labores facilita un estado de ansiedad constante más elevado que anteriormente. De la misma manera, el consumo adicional de tiempo implica, como se ha explicado más arriba, una dificultad añadida para condicionar nuevos discriminativos de cadenas reforzantes en el entorno de su domicilio. La mala relación con su nueva superior también implica una mayor carga aversiva asociada al trabajo.

- Amigas con "su mismo problema": R. tiene un par de amigas que dicen "comprender" su problema y haber pasado por lo mismo. R. encuentra muy agradable el conversar con ellas. Estas conversaciones han dotado de un valor muy reforzante a hablar del problema.

- Sistema inmunológico relativamente débil: independientemente de la carga psicológica que tenga el problema de R., existe una mayor vulnerabilidad a síntomas leves pero molestos como los dolores de cabeza, de estómago, náuseas, etc. Los médicos han descartado otras enfermedades, salvo una ligera anemia.

- Familia del marido y grupos de amigos importantes muy lejos del lugar de residencia: esto obliga a que R. y su marido hagan frecuentemente viajes relativamente largos.

- Función prioritaria de refuerzo: refuerzo negativo. R. realiza muy pocas actividades por el placer que ello pudiese reportarle, dedicándose más bien a "descansar" y a evitar problemas. Esto significa, a grandes rasgos, que el estado de ánimo de R. en el mejor de los casos se mantendrá plano con oscilaciones negativas. En el momento actual, implica un estado de ánimo muy decaído.

Aunque lo lógico es pensar que R. siempre ha tenido una mayor vulnerabilidad a los correlatos fisiológicos de la ansiedad y otras alteraciones, esto parece haberse agravado desde el momento en que comenzó a buscar una explicación psicológica a su problema. Antes de eso, se encontraba mal pero no le daba vueltas a la situación, contentándose con la explicación que ya tenía. En el momento en el que se le sugirió una explicación alternativa comenzó a analizar constantemente su estado y a anticipar los problemas que le generaría caer enferma.

En cuanto a sus dos episodios de ansiedad elevada, debido a la distancia en el tiempo y lo poco detallado de los recuerdos de ambos, no podemos establecer una hipótesis clara. En todo caso, la presentación de los síntomas ansiosos provocó que tanto el salón como el baño se convirtiesen en estímulos condicionados de una respuesta de ansiedad, que se trató de controlar con conductas de escape como abrir la ventana y asomarse o incluso salir a la calle.

La exposición constante al complejo estimular "estar en el salón", sin embargo, ha hecho que pierda gran parte de su potencial ansiógeno, presentándose sólo de forma ocasional los pensamientos relativos a que pueda pasar algo allí cuando ella ya se encuentra mal. La ducha, presumiblemente por el menor tiempo de exposición y la brevedad de la misma (insuficiente para que la curva de ansiedad descienda, provocando una leve incubación), no ha perdido su potencial ansiógeno.

En cuanto al trabajo, hay un ejemplo perfecto de adquisición por parte del entorno de un potencial ansiógeno importante y su posterior eliminación gracias a la exposición continuada: el atraco que sufrió. La poca importancia que ella da a esto en el momento actual puede obedecer a que las verbalizaciones al respecto que ella efectúe tengan una función meramente de llamada de atención en relación con su marido, pero es imposible saberlo con los datos disponibles. 
Por lo demás, el aumento en la presión laboral, teniendo en cuenta que sus problemas de salud comenzaron -o se agravaron- cuando empezó a trabajar, habrá condicionado el trabajo como complejo estimular ansiógeno. El que no haya perdido este potencial puede deberse a muchas cosas, entre ellas el hecho de que se estén administrando constantemente estímulos nuevos pero ansiógenos de forma contingente con el horario de trabajo (exigencias nuevas, comentarios de la jefa) o las constantes verbalizaciones relativas al estrés del trabajo que ella realiza en situaciones no laborales, en las que destaca los aspectos negativos y estresantes de su trabajo.

En lo relativo a los viajes y su reticencia a ir, resulta algo más difícil buscar un origen a su conducta actual. Tal vez lo más parsimonioso sea que, dada la insistencia constante de su marido en ir a su ciudad de origen a vivir por lo insoportable de las condiciones de vida en su ciudad de residencia actual y el que quiera ir siempre allí, el propio hecho de ir a la ciudad de su marido se haya condicionado como aversivo, y esté entrando en juego una estrategia evitativa de afrontamiento.

Actualmente, su estado casi constante de malestar puede explicarse por una mezcla entre el carácter levemente ansiógeno de prácticamente todos los entornos en los que se maneja y su constante autoobservación, ansiógena en sí por el aumento en saliencia de los síntomas observados. Esta autoobservación está sometida a refuerzo variable por el alivio que produce el encontrarse ocasionalmente mejorando. (fig.1)

Por otra parte, el refuerzo social obtenido por hablar de "su problema" aumenta el malestar debido a la carga de ansiedad aparejada a palabras como "enferma", "mala", etc., así como a la prosodia con la que se expresa al respecto (tono cansado, plano, desganado). (fig.2)

Una funcionalidad más del mostrarse enferma es la evitación de otras tareas ansiógenas o condicionadas como negativas, como las anteriormente relativas a la ciudad de su marido. Igual que en la cadena anterior, esta verbalización apareja un malestar añadido. Esta estrategia se da también ante situaciones que puedan provocar una tensión anticipada, como quedar con sus amigas. Incluso, como teoría adicional, el que pueda anticipar que durante la cita con sus amigas (por ejemplo) va a poder "desahogarse", hablar de su problema, con el consiguiente aumento del malestar, sería una repetición de la cadena expuesta en la figura 2, en una versión encubierta y anticipada. (fig.3)

La ducha en su casa se mantiene como ansiógena, como se ha dicho anteriormente, por un proceso de incubación (Eysenck, 1985). Cuando ella se está duchando se dice a si misma que no ocurre nada y que no tiene por qué agobiarse, mientras se autoobserva con atención. Como en los casos anteriores, esta autoobservación tiene en sí misma una capacidad de provocar ansiedad importante que impide el descenso de la ansiedad en la situación de la ducha. Además, ella se da prisa al ducharse, favoreciendo ese proceso de incubación. (fig.4)

Durante los fines de semana, cuando $R$. se pone enferma, se mete en la cama. El momento en el que decide hacerlo es más una cuestión de grado que de modo; es decir, no es la aparición de un síntoma en concreto lo que señala el quedarse en la cama, sino la intensidad de los síntomas en general. En cualquier caso, se queda en la cama "sintiéndose mal", lo que funciona de la misma manera que otras cade-

Figura 1.

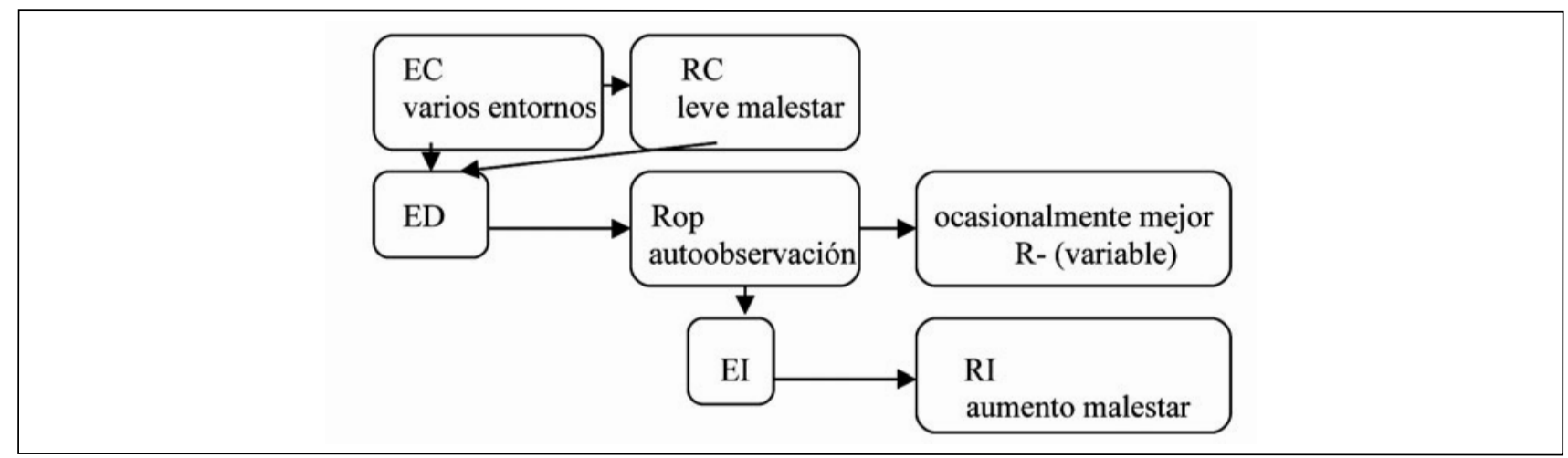


Figura 2.

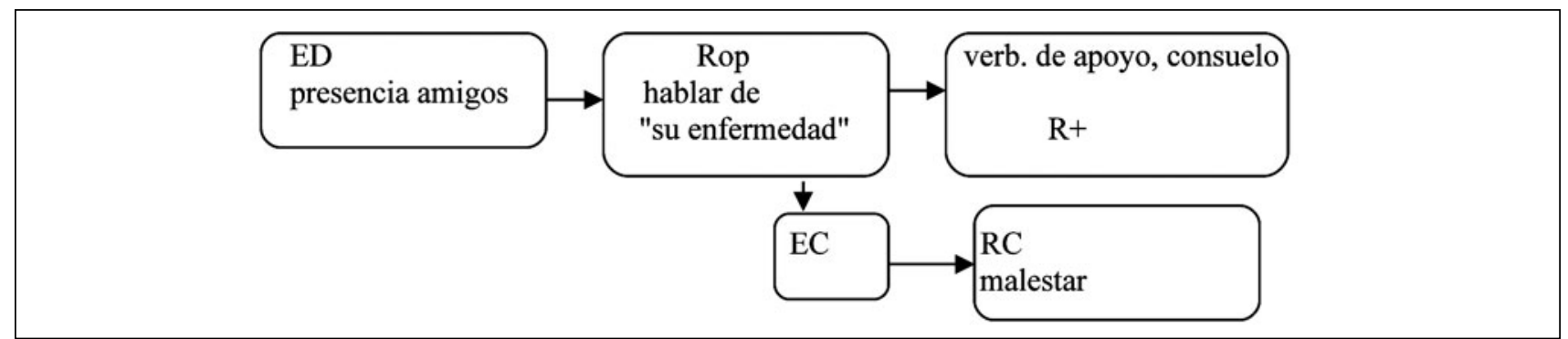

Figura 3.

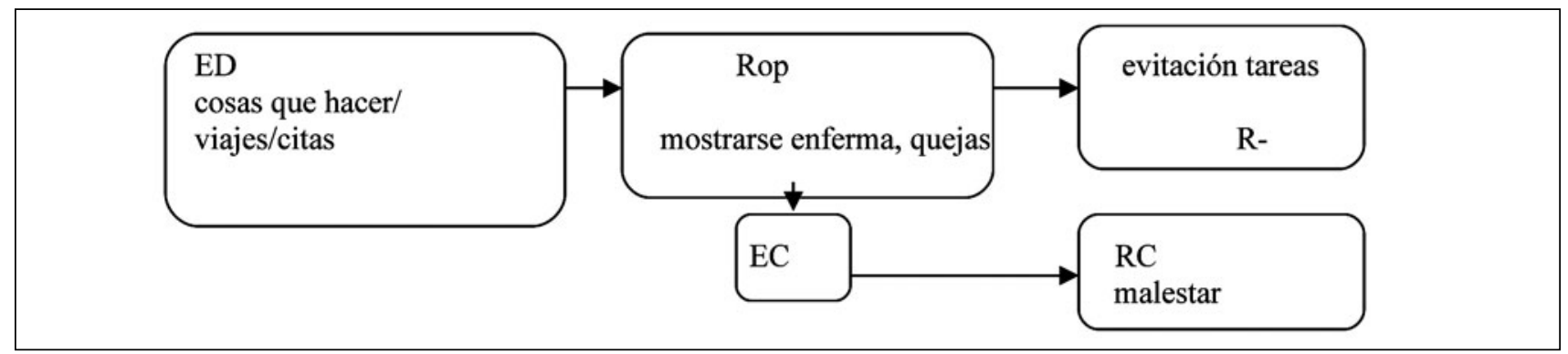

Figura 4.

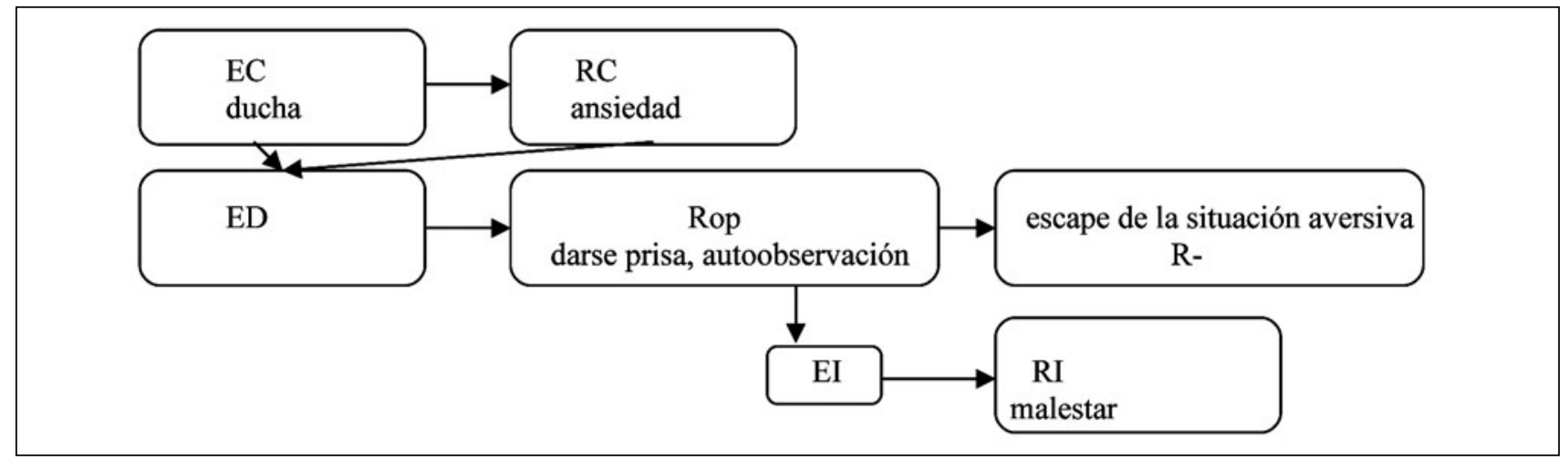

nas señaladas previamente en cuanto a que refuerza una evitación que precisamente por su propia efectividad le hace sentirse peor. Puede ser una forma extrema usada en situaciones en las que otra evitación sería insuficiente para alejarla de los estímulos ansiógenos. (fig. 5)

Teniendo todo lo anterior en cuenta se planteó un único objetivo de tratamiento: eliminar las respuestas de ansiedad y mantener las actividades cotidianas, para lo que tendrá que hacer cosas a pesar de encontrarse mal (que los síntomas dejen de funcionar como discriminativo de evitación o inactividad, $\mathrm{y}$ adquirir discriminativos y respuestas que favorezcan un control no evitativo de las respuestas de ansiedad). Se marcó este objetivo principal dado que es esta evitación la que, como se ha explicado en el análisis funcional, provoca el estado actual de R. Una vez eliminadas las conductas de evitación se favorecerían muchas más oportunidades para aprender nuevas asociaciones y eliminar la autoobserva-

Figura 5.

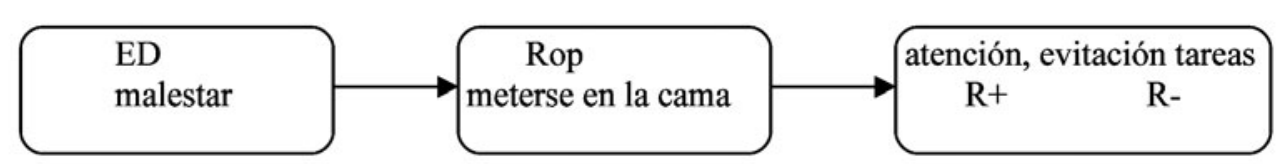


ción como estrategia principal. Esto pasa por dotar a R. de un control mayor sobre sus propias reacciones a la ansiedad, algo que en varias ocasiones durante la evaluación cita como deseable. Se tomó como baremo de la consecución de este objetivo el que R., cuando comente en sesión algún malestar que haya tenido durante la semana, verbalice también tener control (aunque sea relativo) sobre esos síntomas; se tuvieron en cuenta también las verbalizaciones emitidas en sesión que describieran una contingencia natural (fuera de terapia) o un cambio en una de esas contingencias que favoreciera la marcha de la terapia (es decir, sin conductas evitativas y haciendo uso de las habilidades aprendidas en sesión).

Adicionalmente, se propuso como objetivo aumentar el número de actividades reforzantes que R. realizaba en su vida, que en el momento de acudir a sesión eran muy pocas. De esta manera se pretende mejorar el estado de ánimo.

Para el objetivo principal de la terapia se utilizaron las siguientes técnicas (Martin y Pear, 1999; Pérez, 1996b):

- Relajación muscular progresiva (Méndez, Olivares y Quiles, 1998): Se entrenó a R. en el uso de esta técnica para introducirla como una respuesta que compitiera con la autoobservación que ella usaba y que tuviera un efecto relajante sobre el cuerpo. Además, aumentaría el control percibido y real de R. sobre sus propias reacciones corporales.

- Respiración profunda: Con el mismo objetivo que la técnica anterior, se enseñó a R. a usar la respiración profunda. El hacerlo de manera adicional a la relajación muscular responde al hecho de que la respiración es menos conspicua de llevar a cabo, cosa que permitiría a R. usarla tan a menudo como quisiera en su lugar de trabajo.

- Parada de pensamiento con autoinstrucciones y actividades distractoras (Santacreu, 1991): Dada la importancia de la autoobservación y la anticipación de contingencias aversivas en el caso de R., se consideró que era esencial detener la espiral de pensamientos autoevaluadores en la que se veía inmersa de forma bastante frecuente. Así pues, se le instruyó en la técnica de la parada de pensamiento, y se le dieron instrucciones para que si los pensamientos aparecían cuando estaba haciendo alguna cosa que lo permitiera, se centrase en la tarea que tenía entre manos (ver una película, conducir); en caso de no ser posible, debía introducir autoinstrucciones y verbalizaciones que compitieran con aquellas a eliminar, como por ejemplo "esto no me impedirá pasármelo bien".

- Exposición con prevención de respuesta (Echeburúa y Corral, 1993) Una vez R. dominó aceptablemente las técnicas arriba mencionadas y mostró una cierta mejoría, se trabajó con ella el problema de su miedo a la oscuridad y a la sensación de estar en la ducha encerrada. Se le dieron las explicaciones pertinentes y se insistió en lo importante de no escapar de la situación de forma pasiva.

- Control estimular: Se buscó modificar el valor que tenía la presencia de otros como discriminativo de comenzar a hablar de sus problemas, y sustituirlo por la discriminación de conductas alternativas y no compatibles, como hablar de otra cosa o preguntar a la otra persona por sus asuntos. Se puso especial cuidado en que R. comprendiera el valor evocativo de las palabras.

- Programación de actividades reforzantes con el objetivo de mejorar el estado de ánimo. Se instruyó a R. para que programara de antemano las actividades que les gustaría hacer a ella y su marido, de tal forma que no tuvieran que esperar a tener ganas en el momento de hacerlo.

El tratamiento se inicia con la explicación del análisis funcional que $\mathrm{R}$. comprende y acepta, lo cual es buen predictor de su implicación en la terapia. En todo momento cumplió con las tareas y se mostró muy participativa, comentando las dificultades con las que se iba encontrando o incluso anticipándose a las que pudieran ocurrir, lo que fue muy positivo para el proceso.

En algún momento surgen pequeñas dificultades que no le permiten entrenar las técnicas como se había estipulado, pero no dejó de practicarlas en ningún momento. Aún sin controlarlas, ya en sesión verbaliza que sabe que tiene los recursos por si surgiera alguna situación problemática (Froján, Calero, Montaño, 2006).

A partir de la novena sesión se empiezan a distanciar las sesiones cada quince días. A partir de enton- 
ces, no sólo aplica las técnicas, sino que es capaz de anticipar posibles problemas que pudieran surgir y ella misma es quien propone distintas maneras de actuar y además, de una forma muy adecuada.

Dado el avance hasta el momento, se empieza a dedicar más tiempo a conversaciones neutras o positivas, para que la terapia deje de ser discriminativa de hablar sólo de situaciones problema.

En sesión también se trabaja una situación difícil (una cirugía), que formará parte de la generalización de los aprendizajes que se dieron durante la terapia y que se consideró como alta definitiva al cabo de 14 sesiones.

El objetivo de este estudio es comprobar hasta qué punto las verbalizaciones del cliente están sujetas a control por parte del terapeuta. Lo esperable es que fuera así y que se cumplieran las hipótesis iniciales que la literatura al respecto nos lleva a esperar (Froján, 2011).

Al empezar el tratamiento se espera observar una mayor frecuencia de discriminativos por parte del terapeuta para evocar un mayor número de conductas adaptativas a reforzar, sean estas conductas adaptativas verbalizaciones en las que R. afirme su propio control sobre el problema o describa contingencias concretas que redundarán en su beneficio.

Según avance el tratamiento se espera que las verbalizaciones de control generales del cliente disminuyan para pasar a verbalizaciones discriminativas de conductas adaptativas muy concretas. Esto reflejaría el proceso correcto de moldeamiento.

$\mathrm{Al}$ acercarse el alta terapéutica se espera que aumenten las categorías "Otros adaptativos" (ver método) del terapeuta y del cliente, siendo una muestra de que el aprendizaje se ha producido.

En cuanto a las verbalizaciones desadaptativas del cliente, debería haber una reducción significativa según avanza el tratamiento (Froján, Montaño, Calero y Ruiz, 2011). Se puede esperar alguna verbalización desadaptativa en caso de presentarse cualquier situación problemática en la que el cliente no haya podido anticiparse y haya tenido problemas para aplicar las técnicas (caída) y se haya reforzado la conducta en esa situación problemática, aunque estas verbalizaciones desadaptativas no deberían ser seguidas de otra cosa que no fuera lo que podríamos describir como un proceso de moldeamiento de esa respuesta desadaptativa para transformarla en una adaptativa.

\section{Método}

La conducta del terapeuta ha sido analizada a través de grabaciones de las sesiones, después de contar con el consentimiento del cliente.

Se comenzó a registrar a partir de la cuarta sesión, tras la explicación del análisis funcional, ya que es a partir de entonces cuando se puede observar la interacción terapeuta-cliente dirigida hacia la consecución de los objetivos establecidos.

Nuestra hipótesis era que durante la fase de evaluación se daría una mayor frecuencia de discriminativos por parte del terapeuta seguidos de descripciones e información por parte del cliente. Por lo tanto no contamos con el registro de la conducta del terapeuta en este caso, ya que no está orientada a introducir ningún cambio, sino a recopilar la información relevante del caso. Aunque es cierto que se sigue evaluando durante todo el tratamiento para comprobar que se estén aplicando correctamente las técnicas entrenadas en sesión y que se esté obteniendo el resultado deseado, esta evaluación se da más bien integrada en el propio proceso de cambio guiado por la conducta verbal del cliente, por lo que a efectos de este artículo no supone una variable que interfiera.

Dentro de la conducta del terapeuta distinguimos cinco funciones de la conducta verbal, para cuya clasificación nos hemos inspirado en el modelo propuesto por Froján, Montaño, Calero, García, Garzón y Ruiz (2008).

- Función discriminativa: con el fin de evocar determinadas verbalizaciones

- Función de refuerzo: para aumentar la probabilidad de ocurrencia de determinadas verbalizaciones

- Función de castigo: para reducir la probabilidad de ocurrencia de determinadas verbalizaciones

- Función evocativa: para elicitar determinadas respuestas condicionadas

- Otros: información adicional, charla irrelevante. 
La conducta del cliente se ha dividido en dos grandes categorías, cada una de ellas con tres subcategorías.

\section{Verbalizaciones adaptativas}

1.1 Verbalizaciones de control (VC): aquellas verbalizaciones que describen contingencias generales de mejora o posibilidad de controlar el problema.

Ejemplo: "Sé que en cuanto lo entrene estaré bien".

1.2 Discriminativos de conductas adaptativas (EdA): el cliente describe contingencias concretas para la modificación de la conducta problemática.

Ejemplo: "En ese caso permanezco en el lugar hasta que el nivel de ansiedad disminuya".

1.3 Otros (OtA): cualquier verbalización que no pertenezca a las categorías anteriores, pero que facilite la participación activa durante la terapia. Podría ser por ejemplo cualquier tipo de verbalización que compare el estado previo al tratamiento con el estado durante el tratamiento.

Ejemplo: "Yo antes era incapaz de hacer cosas que ahora sí hago".

\section{Verbalizaciones desadaptativas}

2.1 Verbalizaciones de falta de control (VFC): aquellas verbalizaciones que describen con- tingencias generales de "no control" del problema.

Ejemplo: "Voy a estar así toda la vida, yo soy asî".

2.2 Discriminativos de conductas desadaptativas (EdD): el cliente describe contingencias concretas de conductas desadaptativas de tipo evitativas.

Ejemplo: "No voy a poder permanecer en la habitación a oscuras".

2.3 Otros (OtD): cualquier verbalización que no entre en las categorías anteriores, pero que suponga un problema para el buen curso de la terapia.

Ejemplo: "Es que yo soy asî"

\section{Resultados}

Se han clasificado las cadenas funcionales de la siguiente manera:

En las siguientes figuras se presentan los gráficos de las distintas cadenas funcionales registradas durante la observación de las sesiones.

Las cadenas más frecuentes (líneas de mayor grosor) durante el tratamiento psicólogico han sido las siguientes:

- Verbalizaciones de control reforzadas por el terapeuta (1. Ed-VC-R+)

Tabla 1 Cadenas deseables

Verbalizaciones de control reforzadas por el terapeuta

Verbalizaciones discriminativas de conductas adaptativas reforzadas por el terapeuta

Verbalizaciones "otros" adaptativas reforzadas por el terapeuta

Verbalizaciones de falta de control castigadas por el terapeuta

Verbalizaciones discriminativas de conductas desadaptativas castigadas por el terapeuta

Verbalizaciones "otros" desadaptativas castigadas por el terapeuta

Verbalizaciones de control reforzadas por el terapeuta tras haber dado discriminativos adicionales

Verbalizaciones discriminativas de conductas adaptativas reforzadas por el terapeuta tras haber

dado discriminativos adicionales
1. Ed-VC-R+

2. Ed-EdA-R+

3. Ed-OtA-R+

4. Ed-VFC-C

5. Ed-EdD-C

6. Ed-OtD-C

7. Ed-EdD-Ed-VC-R+

8. Ed-EdD-Ed-EdA-R+

Tabla 2 Cadenas no deseables

Verbalizaciones de falta de control reforzadas por el terapeuta

Verbalizaciones discriminativas de conductas desadaptativas reforzadas por el terapeuta

Verbalizaciones "otros" desadaptativas reforzadas por el terapeuta

Verbalizaciones de control castigadas por el terapeuta

Verbalizaciones discriminativas de conductas adaptativas castigadas por el terapeuta

Verbalizaciones "otros" adaptativas castigadas por el terapeuta

Verbalizaciones de control extinguidas por el terapeuta

Verbalizaciones discriminativas de conductas adaptativas extinguidas por el terapeuta

Verbalizaciones "otros" adaptativas extinguidas por el terapeuta
1. Ed-VFC-R+

2. Ed-EdD-R+

3. Ed-OtD-R+

4. Ed-VC-C

5. Ed-EdA-C

6. Ed-OtA-C

7. Ed-VC-X

8. Ed-EdA-X

9. Ed-OtA-X 
Gráfico 1.

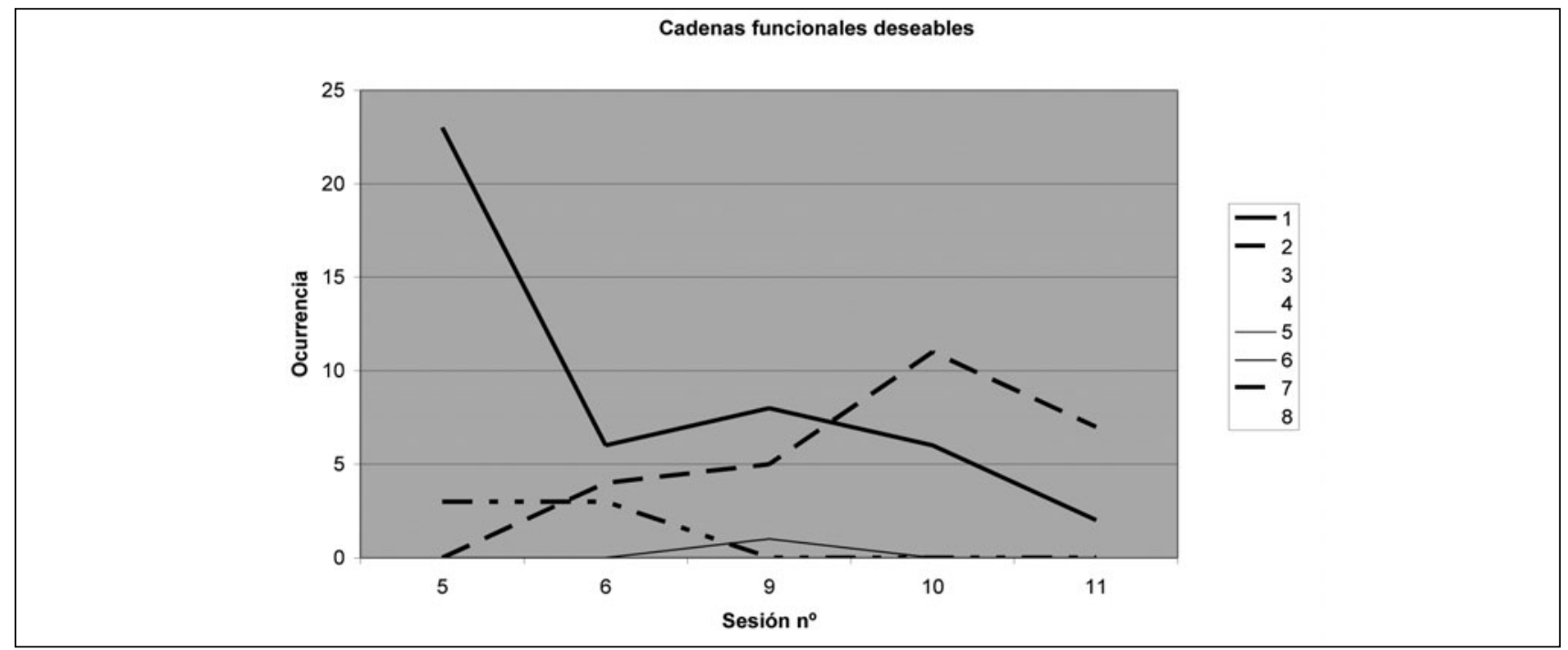

Gráfico 2.

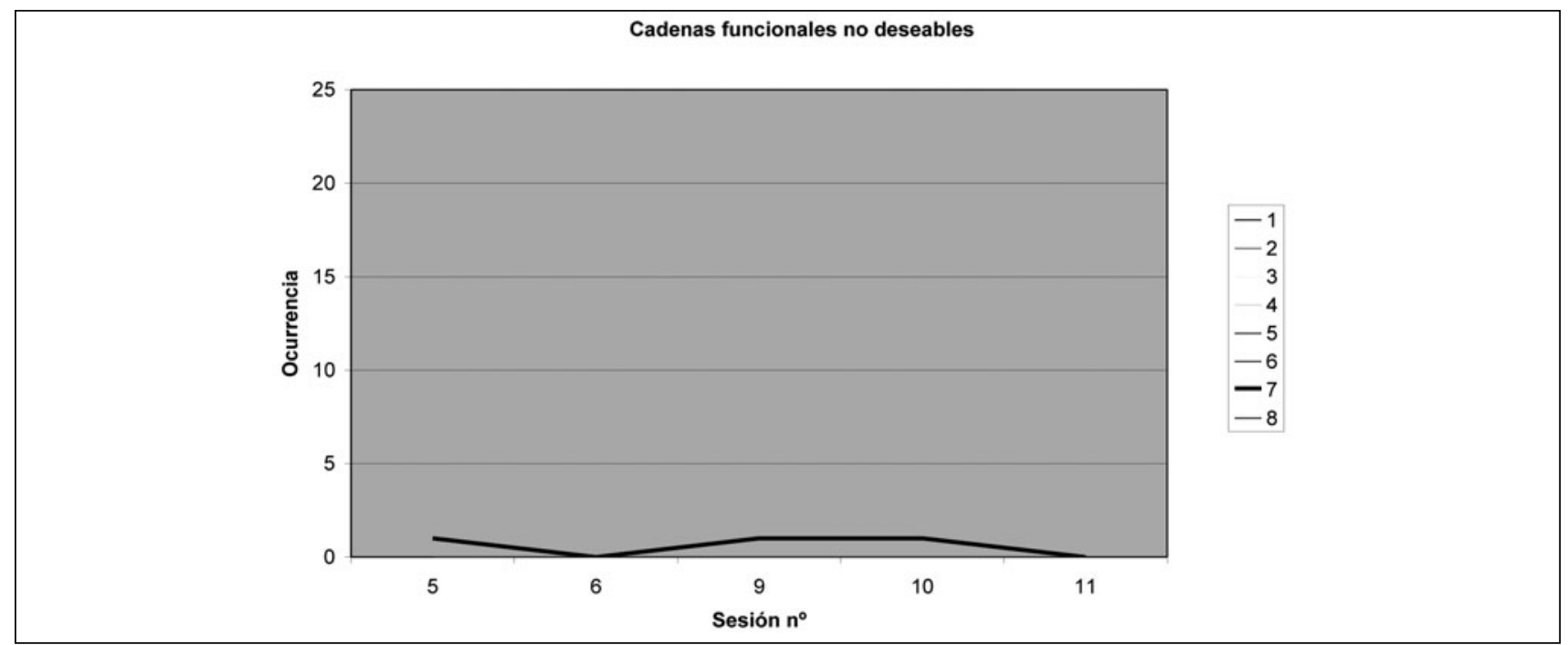

Gráfico 3.

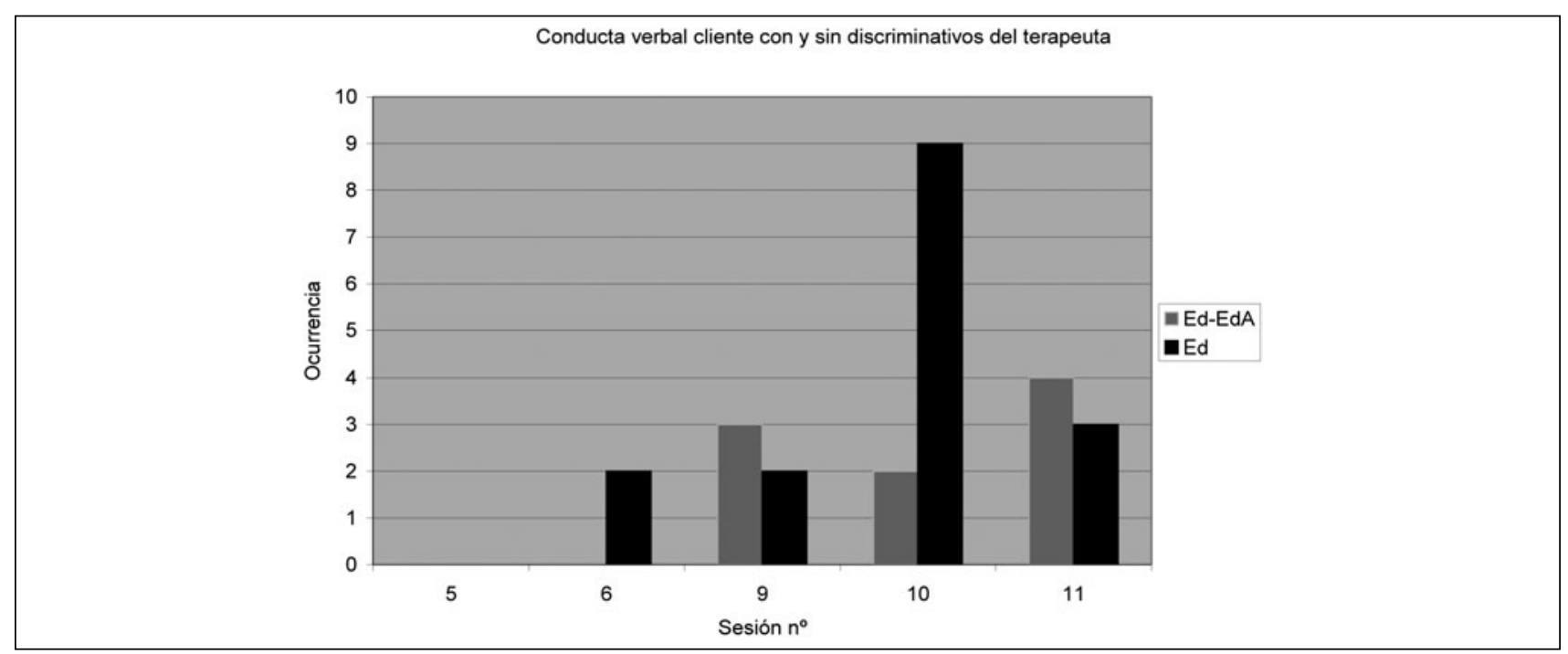


- Verbalizaciones discriminativas de conductas adaptativas reforzadas por el terapeuta (2. EdEdA-R+)

- Verbalizaciones de control reforzadas por el terapeuta tras haber dado discriminativos adicionales (7. Ed-EdD-Ed-VC-R+)

Se puede observar cómo las verbalizaciones de control sobre el problema son más frecuentes al inicio del tratamiento. Según éste avanza, estas verbalizaciones empiezan a disminuir para pasar a aumentar aquellas que son discriminativas de conductas adaptativas.

En cuanto a las cadenas funcionales no deseables sólo aparecen 3 de ellas.

Cada una de estas cadenas aparece una única vez $\mathrm{y}$ de forma aislada en las sesiones 5,9 y 10 .

El número de cadenas adaptativas asciende a 87 , mientras que las no deseables sólo llegan a 3 .

Por último, se muestra un gráfico que analiza el número de cadenas en las que las respuestas discriminativas de conductas adaptativas del cliente vienen precedidas por un discriminativo del terapeuta o si, por el contrario, es el propio cliente quien lo verbaliza sin necesidad de que el terapeuta los plantee.

\section{Discusión y conclusiones}

Con los resultados previamente expuestos podemos considerar que nuestras hipótesis iniciales se cumplen. Esto nos lleva a una reflexión de mayor alcance acerca de la naturaleza de la interacción clínica y la relevancia de la metodología observacional para su estudio. Es importante comprender las limitaciones que esto significa, y que, pese a que -en interés de la brevedad y de no ser farragosos- hablamos de que "se ha dado un proceso de moldeamiento" o "se ha reforzado o castigado tal conducta", lo que se quiere decir con eso es que la conducta en concreto varía como variaría en caso de estar sometida a un proceso de moldeamiento, refuerzo, castigo o cualquier otro paradigma de modificación de conducta (Froján, Montaño y Calero, 2006).

Evidentemente, la literatura clínica no puede cumplir los mismos criterios que la experimental; los controles necesarios para ello convertirían la actuación terapéutica en artificial y probablemente esto redundaría en una atención deficiente a lo que debe ser nuestra primera preocupación: el bienestar del cliente. El carácter obligadamente aplicado de nuestra disciplina nos obliga a menudo a un cierto apresuramiento a la hora de aplicar lo que sabemos en la práctica clínica, en ocasiones despreciando la investigación por considerarla demasiado etérea o tan envuelta en controles que su validez ecológica queda irremediablemente menoscabada. Esta separación, tanto más grave cuanto menos sometida a control está la praxis clínica, debe desaparecer poco a poco. La literatura clínica y la de investigación deben retroalimentarse mutuamente, supliendo cada una las carencias de la otra. Una forma de conseguir esto desde la literatura clínica es aumentar la exigencia en cuanto a la descripción de detalles y procedimientos. Obviamente, jamás podrá considerarse completamente replicable un caso clínico; sin embargo tener una información clara y completa sobre lo que el terapeuta hizo, más allá de la simple enumeración de las técnicas usadas, es a nuestro juicio esencial (Barnes-Holmes y Hayes, 2002; Barnes-Holmes, 2005; Frojan, Montaño y Calero, 2010). Muy a menudo se incluye en los casos clínicos una información tremendamente detallada acerca de las modificaciones hechas a los protocolos estándar de tratamiento para adaptarlos a las variables propias del caso y/o del cliente. Esta información es, indudablemente, de gran valor. Sin embargo se puede incurrir (y se incurre a menudo) en el error de obviar la información detallada sobre cómo el terapeuta implementa esas técnicas, cómo instruye al cliente para hacerlas o cómo responde ante los imprevistos. Es cierto que ocasionalmente encontramos descripciones de estos sucesos, pero muy rara vez estas descripciones incluyen un análisis funcional de la conducta del terapeuta en sesión con el cliente. Es ésta la parte esencial, a nuestro parecer, para conocer realmente la intervención realizada por el profesional y poder -en la medida de lo posible- aplicar en otros casos similares la misma, aumentando así la eficacia y eficiencia del tratamiento (Garfield y Bergin, 1994; APA Task Force on Evidence-Based Practice, 2006). Al fin y al cabo, en todo contexto se están dando constantemente interacciones de aprendizaje (Freixa, 2003), y la consulta es un contexto más, con dos salveda- 
des importantes: en él se habla de cosas problemáticas para el cliente y es un entorno en el que el terapeuta controla las contingencias a administrar. Es de la correcta administración de estas contingencias de la que depende el buen término del tratamiento.

Centrándonos más en el caso estudiado, podría encajar a grandes rasgos en lo que se suele denominar "problemas de ansiedad". El éxito y la facilidad con la que estos problemas se tratan de manera regular en el entorno terapéutico favorece que sean abordados en la literatura clínica centrándose fundamentalmente en las técnicas usadas y las adaptaciones hechas para ajustarse al caso concreto. Se prima la información sobre las técnicas que el cliente debe aplicar fuera de sesión por encima de las realizadas durante el tiempo de consulta. La conducta verbal del terapeuta queda, pues, como una variable enmascarada en el éxito o fracaso del tratamiento; una variable que puede llegar a ser muy importante, si no la más importante (por ejemplo: Hamilton, 1988; Varela, 2008; Roca, 2006; Kazdin, 2008). Al fin y al cabo, el instrumento que el terapeuta usa en todo momento desde que ve por primera vez al cliente es su propia conducta verbal (Catania, 1998), y ésta no se emplea de forma aleatoria: la conducta verbal tiene distintas funciones cuyo adecuado manejo y administración serán lo que marcarán un cambio en la conducta del cliente (Cruzado, Labrador y Muñoz, 1993; Froján et al., 2011; Follette, Naugle y Callaghan, 1996; Hamilton, 1988; Pérez, 1998; Rosenfarb, 1992). De forma más específica se pueden estar planteando las siguientes cuestiones: ¿Cómo consigue el terapeuta que el cliente ponga en práctica fuera de sesión lo que se le enseña? ¿Cómo lo hace para que, por ejemplo, la perspectiva de la ansiedad que se sufrirá durante una exposición no impida que ésta se lleve a cabo? ¿De qué manera el terapeuta administra consecuentes verbales para maximizar el efecto terapéutico? ¿Cómo se hace, en definitiva, para que el cliente "quiera" hacer lo que el terapeuta le dice que haga? (Skinner, 1966; Skinner, 1969; Pérez, 1996a; Pérez, 2004; Pérez, 2006). En nuestra opinión, la única forma de comprender el cambio conductual del cliente es analizarlo funcionalmente y, dado que las contingencias que lo moldean hasta parecerse más al comportamiento que el propio cliente ha marcado como objetivo no son aleatorias, sino que están sujetas a una planificación por parte del terapeuta, un análisis funcional de la conducta de este último es imprescindible para comprender realmente cómo se ha producido ese cambio terapéutico (Cruzado, Labrador y Muñoz, 1993; Vila, 1997; Garfield y Bergin, 1994; Hill, Nutt y Jackson, 1994; Follette, Naugle y Callaghan, 1996; Terraz, de Roten, de Roten, Drapeau y Despland, 2004; Wilson y Blackledge, 2000; Rosenfarb, 1992).

El hecho de que las verbalizaciones discriminativas de conductas adaptativas aumenten y las verbalizaciones de control disminuyan conforme avanza el tratamiento, concuerda con los datos esperados, ya que el cliente, al haber tenido más ensayos de práctica de las conductas adaptativas, es capaz de describir las contingencias exactas en sesión.

En cuanto a las cadenas no deseables (por lo antiterapéuticas) emitidas por el terapeuta, hay que destacar que las cadenas que le siguen son deseables (es decir, favorecen el moldeamiento en la dirección adecuada del tratamiento). Aunque esto no altera su condición de no deseables, sí atenúa el posible efecto contraproducente que pudieran tener.

También se han querido destacar las cadenas funcionales (7. Ed-EdD-Ed-VC-R+), que aparecen en las sesiones 5 y 6 ( 3 veces en cada una de ellas). El que aparezcan en las sesiones 5 y 6 se puede explicar a través del moldeamiento que el terapeuta hace durante las sesiones de entrenamiento de nuevas habilidades, cuando el cliente puede necesitar el refuerzo de aproximaciones sucesivas a la conducta objetivo. Evidentemente, durante la sesión el terapeuta presentará muchos discriminativos que favorecerán que $\mathrm{R}$. dé las respuestas deseadas (para que puedan ser reforzadas) o erróneas (para que puedan ser moldeadas, castigadas o extinguidas) (Barraca, 2009).

En lo que se refiere a las verbalizaciones que funcionan como discriminativos de conductas adaptativas emitidas por el cliente, como se recordará, se ha tenido en cuenta si han sido discriminadas por el terapeuta o no. Lo deseable sería que según avanzara el tratamiento, aumentara el número de ocasiones en las que R. emitiera estas verbalizaciones sin necesidad de un discriminativo administrado por el terapeuta. Esto supondría un buen pronóstico en cuanto a la generali- 
zación que R. haya hecho de lo aprendido en sesión; al fin y al cabo, fuera del contexto terapéutico no estará el psicólogo como elemento de control externo. Que ella misma los administre es un marcador de mejora importante. Hay que destacar que en la sesión 11 los datos no son favorables a lo esperado en un caso en curso de mejora como era este; no obstante, esto puede explicarse por el hecho de que la sesión duró tan solo media hora, en lugar de los 60 minutos de las previas. Es de esperar que, de haber durado lo mismo, las variaciones fueran en la misma dirección que en las otras, aunque evidentemente no hay forma de comprobarlo.

Se podría decir, por lo tanto, que la conducta verbal del terapeuta (Dymond, O'Hora, Whelan, O'Donovan, 2006) es decisiva en la mejoría por parte del cliente, cosa que a pesar de ser evidente (al fin y al cabo, es él quien debe instruirle), es frecuente que se pase por alto. Aunque muchas veces no se dé la propia situación problemática y la técnica específica en sesión, sí se han dado los discriminativos de forma clara, cosa que facilita la generalización, y en muchas ocasiones las verbalizaciones se modelan para asegurar el aumento de proba- bilidad de puesta en marcha de las técnicas aprendidas.

Somos conscientes de que el alcance de este artículo puede parecer inicialmente demasiado limitado, incluso carente de interés o poco vistoso; sin embargo, nos gustaría recordar que los imperativos de la ciencia son describir, explicar, predecir y controlar, y que una buena descripción, aunque pueda parecer en un principio algo limitado, es la base sobre la que se edifica todo lo demás.

Por último, consideramos que, aunque el caso en sí no reviste mayor dificultad técnica -debido a la relativa simplicidad del asunto y a la colaboración del cliente a la hora de poner en marcha las técnicas-, el análisis funcional de la conducta verbal del terapeuta da una información tremendamente valiosa sobre lo que realmente está ocurriendo en sesión. Sería interesante realizar el mismo trabajo sobre casos más complejos o de más larga duración, con altibajos de seguimiento por parte del cliente, para comprobar qué variables comportamentales pueden estar influyendo en esa oscilación; generar un volumen de datos al respecto, podría llevarnos a conclusiones verdaderamente relevantes una vez tomados en su conjunto.

\section{Extended Summary}

The paper discusses a typical anxiety case. A woman, R., 33 years old, describes different problematic situations in which she shows dysfunctional behaviours that aggravate the problem. During the assessment process, she described her problem, emphasizing physical discomfort such as sickness, fever, headaches and a feeling of nervousness associated with specific situations. She had tried different treatments, but organic problems were dismissed after numerous medical tests.

$R$. identifies the beginning of the problem with a "panic attack" she suffered at home, after arriving from work. Later on, she started to consider the possibility of a psychological problem and the symptoms began to get worse, matching up with the uncertainty about the origin and continuance of the problem.

As a result, she adapted her lifestyle to her problem. Simultaneously, her fear of darkness and the nervousness she felt while having her daily shower worsened. She was also feeling uncomfortable at her workplace.

After compiling the relevant information, a functional analysis was made. Due to the origin of the problem, it was hypothesized that her alternative explanations of her situation and worries were in fact related to the worsening of the symptoms.

There were also specific situations at home that function as conditioned stimuli of anxiety conditioned responses.

The continuance of the problem is due to her constant observation of the symptoms, increasing her feeling of discomfort. It is important to emphasize the function of her complaining utterances, more so as they are reinforced by her friends and family. Simultaneously, her "illness" enables her to avoid different activities previously conditioned as anxiety-triggering.

The therapeutic objectives were aimed toward 
maintaining her activity regardless of her physical symptoms.

The techniques used in the treatment were progressive muscular relaxation, controlled breathing, thought-stopping techniques combined with selfinstructions and distracting activities, exposure and response prevention, stimulus control and a program of gratifying activities.

The aim of this article is to emphasize the importance of the therapist's verbal behaviour and its influence on the patient's behaviour in a therapeutic process. The therapist's verbal behaviour has different functions oriented to enhancing adaptive learning, so the patient can modify the problematic situation.

In the opinion of the authors, the functional analysis of the verbal behaviour of the therapist is crucial to enhancing the success of the treatment. Different functions for the utterances of the clinician during the sessions were identified. These were discriminative utterances, reinforcement utterances, punishment utterances, evocative utterances and others (not included in the above categories).

In addition, the utterances of the patient were classified according to two broad categories: adaptive and non-adaptive utterances.

The first includes control utterances, discriminative utterances that enable adaptive behaviours and others.

The second one includes non-control utterances, discriminative utterances that enable non-adaptive behaviours and others.

After explaining the functional analysis to the patient, the learning process starts. Different behaviour sequences were divided in two categories:

The first is called "therapeutic sequences". It includes the possible combinations of sequences for a successful learning process, such as reinforcing control utterances or punishing the non-controlled utterances of the patient.

The second category is called "non-therapeutic sequences". It includes the possible combinations that would not enable a learning process making progress toward the therapeutic objectives.

After coding the results, three most frequent "therapeutic sequences" were identified in the treatment process. The first one was named "Control utterances being reinforced by the therapist". This sequence showed a progressive decrease during the treatment.
The second most frequent sequence was named "Discriminative utterances enabling adaptive behaviours being reinforced by the therapist". There was a progressive increase in this case during the learning process.

Finally, the third most frequent sequence found by the authors is the category named "Control utterances being reinforced after adding discriminatives". This sequence showed a rapid decrease before disappearing all together.

The results show the importance of the therapist's verbal behaviour in the enabling of the learning process of the patient. At the beginning of the treatment the patient depends on the therapist, but as advances are made in the learning of techniques, the patient shows a generalization of the instructions to new situations and is able to describe in more detail the guideline offered by the therapist.

There were 87 "therapeutic sequences" versus 3 "non-therapeutic sequences".

Finally, the authors emphasize the results showing how the patient reduces her dependence on the therapist by the end of the learning process. The number of discriminatives given by the therapist decreases as the patient expresses them by herself. This is a good predictor of the learning generalization.

As a final conclusion, the authors consider the therapy as a learning process guided by the therapist; more specifically, it is a verbal shaping process. However, this process is not random, since the clinician has to analyze the function of each of his verbal utterances during the interaction in order to guarantee favourable results.

\section{Agradecimientos}

A los autores les gustaría agradecer especialmente la colaboración del Instituto Terapéutico de Madrid (ITEMA).

\section{Referencias}

APA Task Force on Evidence-Based Practice (2006). Evidence-based practice in psychology. American Psychologist, 61, 271-285. 
Barnes-Holmes, D. (2005). Behavioral pragmatism is a-ontological, not antirealist: a reply to Tonneau. Behavior and Philosophy, 33, 67-79

Barnes-Holmes, D. y Hayes, S. C. (2002). Relational frame theory is a behaviour analytic account. Is Tonneau's? European Journal of Behavior Analysis, 3, 87-94.

Barraca, J. (2009) Habilidades Clínicas en la Terapia Conductual Tercera Generación. Clínica y Salud vol. 20, n. 2, pp. 109-117.

Catania, A. C. (1998). The taxonomy of verbal behaviour. En K. A. Lattal y M. Perone (Ed.), Handbook of research methods in human operant behavior (pp. 405-433). Nueva York: Plenum Press.

Cruzado, J. A., Labrador, F. J. y Muñoz, M. (1993 en el texto aparece 1996). Introducción a la modificación y terapia de conducta. En F.J. Labrador, J.A. Cruzado y M. Muñoz (Eds.), Manual de técnicas de modificación y terapia de conducta (pp. 31-46). Madrid: Pirámide.

Dymond, S., O'Hora, D., Whelan, R. y O'Donovan, A. (2006). Citation analysis of Skinner's Verbal Behavior: 1984-2004. The Behavior Analyst, 29, 75-88.

Echeburúa, E.; y Corral, P. (1993). Técnicas de exposición: variantes y aplicaciones. En Labrador, F. J.; Cruzado, J. A. y Muñoz, M (dirs): Manual de técnicas de modificación y terapia de conducta. Madrid: Pirámide.

Eysenck, H. J. (1985): The incubation theory of fear/anxiety. Theoretical foundations of behaviour therapy.

Follette, W. C., Naugle, A. E., y Callaghan, G. M. (1996). A radical behavioural understanding of the therapeutic relationship in effecting change. Behavior Therapy, 27, 623-641.

Freixa, E. (2003). ¿Qué es conducta? International Journal of Clinical and Health Psychology, 3, 595-613.

Froján, M. X. (2011). ¿Por qué funcionan los tratamientos psicológicos? Clínica y salud, 22, 201-204. doi: http://dx.doi.org/10.5093/cl2011v22n3a1

Froján, M. X., Calero, A., y Montaño, M. (2006). Procesos de aprendizaje en las técnicas de reestructuración semántica. Análisis y Modificación de Conducta, 32(143), 287-305.
Froján, M. X., Montaño, M. y Calero, A. (2006). ¿Por qué la gente cambia en terapia? Un estudio preliminar. Psicothema, 18, 797-803.

Froján, M. X., Montaño, M., Calero, A., García, A., Garzón, A. y Ruiz, E. (2008). Sistema de categorización de la conducta verbal del terapeuta. Psicothema, 20, 603-609.

Froján, M. X., Montaño, M. y Calero, A. (2010). Therapist' verbal behavior analysis: A descriptive approach to psychotherapeutic phenomenon. Spanish Journal of Psychology, 13, 914926.

Froján, M. X., Montaño, M., Calero, A. y Ruiz, E. (2011). Aproximación al estudio funcional de la interacción verbal entre terapeuta y cliente durante el proceso terapéutico. Clínica y Salud, 22, 6985. doi: http://dx.doi.org/10.5093/cl2011v22n1a5

Garfield, S. L. y Bergin, A. E. (1994). Introduction and historical overview. En A. E. Bergin y S. L. Garfield (Eds.), Handbook of psychotherapy and behavior change ( $4^{\mathrm{a}}$ ed., pp. 3-18). Nueva York: Wiley.

Hamilton, S. A. (1988). Behavioral formulations of verbal behaviour in psychotherapy. Clinical Psychology Review, 8, 181-193.

Hill, C. E., Nutt, E. A. y Jackson, S. (1994). Trends in psychotherapy process research: samples, measures, researchers and classic publications. Journal of Counseling Psychology, 41(3), 364377.

Kazdin, A. E. (2008). Evidence-based treatment and practice. New opportunities to bridge clinical research and practice, enhance the knowledge base, and improve patient care. American Psychologist, 63(3), 146-159.

Martin, G y Pear, J. (1999). Modificación de conducta. Qué es y cómo aplicarla. Madrid: Prentice Hall.

Méndez, F. X., Olivares, J. y Quiles, M. J. (1998). Técnicas de relajación y respiración. En J. Olivares y F. X. Méndez, F. X, Técnicas de modificación de conducta, Madrid: Biblioteca nueva.

Pérez, M. (1996a). La psicoterapia desde el punto de vista conductista. Madrid: Biblioteca Nueva.

Pérez, M. (1996b). Tratamientos psicológicos. Madrid: Universitas. 
Pérez, M. (1998). La psicología clínica desde el punto de vista contextual. Papeles del Psicólogo, 69, 25-40.

Pérez, M. (2004). Contingencia y drama. La psicología según el conductismo. Madrid: Minerva Ediciones.

Pérez, M. (2006). La terapia de conducta de tercera generación. EduPsykhé: Revista de Psicología y Psicopedagogía, 5(2), 159-172.

Roca, J. (2006). Psicología. Una introducción teórica. Girona: Documenta Universitaria.

Rosenfarb, I. S. (1992). A behaviour analytic interpretation of the therapeutic relationship. Psychological Record, 42(3), 341-354.

Santacreu, J. (1991). El entrenamiento en autoinstrucciones. En V.E. Caballo, Manual de técnicas de terapia y modificación de conducta. Madrid: Siglo XXI.

Skinner, B. F. (1966). An operant analysis of problem solving. En B. Kleinmuntz (Ed.), Problem solving: Research, method and theory (pp. 225257). Nueva York: Wiley.

Skinner, B. F. (1969). Contingencies of reinforcement: A theoretical analysis. Nueva York: Appleton-Century-Crofts.

Terraz, O., de Roten, Y., de Roten, F. C., Drapeau, M., y Despland, J.N. (2004). Sequential therapist interventions and therapeutic alliance: a pilot study. Schweizer Archiv für Neurologie und Psychiatre, 155(3), 111-117.

Varela, J. A. (2008). Conceptos básicos del interconductismo. México: Universidad de Guadalajara.

Vila, J. (1997). Los efectos de la terapia de conducta. Revista de Psicología General y Aplicada, 50, 503-511.

Wilson, K. G. y Blackledge, J. T. (2000). Recent developments in the behavioral analysis of language: making sense of clinical phenomena. En M. J. Dougher (Ed.): Clinical behavior analysis.

Artículo Recibido: 10/06/2011

Revisión Recibida: 29/12/2011

Aceptado: 09/01/2012 